\title{
A REPRESENTATION THEOREM AND APPLICATIONS TO TOPOLOGICAL GROUPS
}

\author{
BY \\ J.-M. BELLEY ${ }^{1}$
}

\begin{abstract}
We show that, given a set $S$ dense in a compact Hausdorff space $X$ and a complex-valued bounded linear functional $\Lambda$ on the space $C(X)$ of continuous complex-valued functions on $X$ with uniform norm, there exist an algebra $\mathcal{Q}$ of sets in $S$ and a unique bounded finitely additive set function $\mu: \mathbb{Q} \rightarrow \mathbf{C}$ which is inner and outer regular with respect to the zero and cozero sets respectively and such that $\int_{S} f \mid S d \mu$ exists and is equal to $\Lambda(f)$ for all $f \in C(X)$. In the context of topological groups, this theorem permits us to obtain (1) a concrete representation theorem for bounded complex-valued linear functionals on the space of almost periodic functions with uniform norm, (2) a representation theorem for (not necessarily continuous) positive definite functions, (3) a characterization of the space $B$ of finite linear combinations of positive definite functions, and (4) a necessary and sufficient condition to have a linear transformation from $B$ to $B$.
\end{abstract}

\section{Introduction.}

1.1. In a paper of Hewitt [4] an attempt was made at obtaining a concrete representation theorem for bounded complex-valued linear functionals defined on the space of continuous almost periodic functions on the real line. Though the problem was not solved satisfactorily (see the footnote on p. 379 in [2]), we were inspired by the methods of that paper to obtain a representation theorem for bounded linear functionals on subspaces of continuous functions defined on sets more general than locally compact Abelian groups. Though a general approach to representation theorems has been developed in recent years (see [3], [10], and [16]), we have found that, to obtain a complex-valued finitely additive set function representing the linear functional, which is inner and outer regular with respect to the zero and cozero sets respectively, the method of Loomis [9, p. 169] to generate an algebra of sets (on which is defined the set function) by means of the linear functional in question was our best approach. In the special case of a topological group, it is this algebra, which depends on the linear functional defined on the almost periodic functions, that makes the difference between our results and those of Hewitt.

We wish to acknowledge valuable conversations with S. Kakutani, which lead to $\$ 2$ in a context more general than that of topological groups.

Received by the editors September 24, 1979.

AMS (MOS) subject classifications (1970). Primary 43A05, 28A25; Secondary 43A25, 43A35, 43A60.

Key words and phrases. Algebras of sets, finitely additive set functions, integral representation of linear functionals, Bohr compactification, almost periodic functions, positive definite functions, FourierStieltjes transforms.

${ }^{1}$ This research was supported by grants from the Natural Sciences and Engineering Research Council, Canada and the Ministry of Education of Quebec. 


\section{A representation theorem on spaces of continuous functions.}

2.1. Let $X$ be a topological space. By a measure on the Borel subsets $\mathscr{B}(X)$ of $X$ we mean a complex-valued (and so finite) countably additive set function on $\mathscr{B}(X)$. Let $C(X)$ and $C_{b}(X)$ denote the continuous and the bounded continuous complex-valued functions on $X$ respectively. Clearly, if $\mu$ is a measure on $\mathscr{B}(X)$, then any $f \in C_{b}(X)$ is integrable with respect to $\mu$ and so the set $\{\omega \in C$ : $\left.\mu\left(f^{-1}(\omega)\right) \neq 0\right\}$ is at most countable. Let $|\mu|$ denote the total variation measure on $\mathscr{B}(X)$ associated with $\mu$. Given an algebra of sets $\mathscr{Q} \subset \mathscr{B}(X)$, let $\mathbb{Q}_{\mu}$ be the algebra of sets $E \in \mathbb{Q}$ such that $|\mu|(\bar{E} \backslash \dot{E})=0$. If $A, B \in \mathbb{Q}_{\mu}$ and if $A \cap S=B \cap S$ for some set $S$ dense in $X$, then $|\mu|(A \triangle B)=0$. This is easily shown from the inclusion

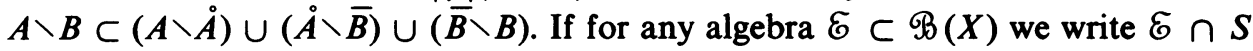
for $\{E \cap S: E \in \mathcal{E}\}$ then $\mathbb{Q}_{\mu} \cap S$ is an algebra of subsets of $S$ such that the set function $\mu_{S}: \mathbb{Q}_{\mu} \cap S \rightarrow \mathbf{C}$ given by $\mu_{S}(E \cap S)=\mu(E)\left(E \in \mathbb{Q}_{\mu}\right)$ is a well-defined bounded finitely additive set function. If $\left|\mu_{S}\right|$ is the total variation of $\mu_{S}$ then clearly $|\mu|(E)=\left|\mu_{S}\right|(E \cap S)=|\mu|_{S}(E \cap S)$ for all $E \in \mathbb{Q}_{\mu}$.

2.2 Definition. Given a set $Y$ and an algebra $\mathcal{E}$ of subsets of $Y$, a function $f$ : $Y \rightarrow \mathbf{C}$ is said to be $\mathcal{E}$-continuous if, given $\varepsilon>0$, there exists a finite partition of $\mathbf{C}$ into rectangles $\left(E_{1}, \ldots, E_{n}\right)$ with $f^{-1}\left(E_{i}\right) \in \mathcal{E}$ and $|x-y|<\varepsilon$ for all $x, y \in E_{i} \cap$ $f(Y)(i=1, \ldots, n)$. We write $C(Y, \mathcal{E})$ for the class of $\mathcal{E}$-continuous functions on $Y$.

2.3 REMARKS. (1) In the context of the definition above, a necessary condition for a function $f: Y \rightarrow \mathrm{C}$ to be $\mathcal{E}$-continuous is that $\int_{Y} f d \nu$ exists for every bounded finitely additive set function $\nu$ on $\mathcal{E}[1$, p. 293], where the integral is defined by the usual Moore-Smith method ([11, pp. 183-191], [15, pp. 401-404]) or equivalently [7] by the Dunford-Schwartz method [2, pp. 101-125]. Note that our definition of $\mathcal{E}$-continuity is more restrictive than the one usually given $[1$, p. 293].

(2) Let $\mathscr{A} \subset \mathscr{B}(X)$ be an algebra of subsets of the topological space $X$ such that each $f \in C_{b}(X)$ is $\mathbb{Q}$-continuous. For example, $\mathbb{Q}=\mathscr{B}(X)$ is such an algebra. Given a complex-valued measure $\mu$ on $Q$ and a set $S$ dense in $X$ such that $f \mid S$ is $\mathbb{Q}_{\mu} \cap S$-continuous for all $f \in C_{b}(X)$, then $\int_{S} f \mid S d \mu_{S}=\int_{X} f d \mu$.

2.4. Let $M R(\mathscr{B}(X))$ denote the space of complex-valued measures $\nu$ on $\mathscr{B}(X)$ which are such that, given $\varepsilon>0$ and $E \in \mathscr{B}(X)$, there is a closed set $C$ and an open set $U$ in $X$ such that $C \subset E \subset U$ and $|\nu|(U \backslash C)<\varepsilon$. Given an algebra $\mathcal{E} \subset \mathscr{B}(X)$, a finitely additive set function $\mu: \mathcal{E} \rightarrow C$ will be said to be regular on $\mathcal{E}$ if, given $\varepsilon>0$ and $E \in \mathcal{E}$, there exist constants $\alpha, \beta>0$ and real-valued functions $f, g \in C_{b}(X)$ such that the sets $K=\{x \in X: f(x)<\alpha\}$ and $V=\{x \in$ $X: g(x)<\beta\}$ have the properties (i) $K, V \in \mathcal{E}$, (ii) $K \subset E \subset V$ and (iii) $|\mu|(V \backslash K)<\varepsilon$.

2.5 Lemma. Given a normal space $X$ and $\mu \in M R(\mathscr{B}(X))$, let $\mathbb{Q} \subset \mathscr{B}(X)$ be an algebra for which each $f \in C_{b}(X)$ is $Q_{\mu}$-continuous. If $S$ is dense in $X$, then $\mu_{S}$ is regular on $\mathbb{Q}_{\mu} \cap S$ and $\mathbb{Q}_{\mu} \cap S$ consists of all $E \in \mathbb{Q} \cap S$ such that

$$
\inf \left\{\int_{S}(h|S-g| S) d\left|\mu_{S}\right|: g\left|S \leqslant \chi_{E} \leqslant h\right| S ; g, h \in C_{b}(X)\right\}=0
$$

where $\chi_{E}$ is the characteristic function for $E$. 
Proof. Since $\mu \in M R(\mathscr{B}(X))$, given $\varepsilon>0$ and $E \in \mathbb{Q}_{\mu}$, there exist a closed set $C$ and an open set $U$ in $X$ such that $C \subset \stackrel{\circ}{E} \subset E \subset \bar{E} \subset U,|\mu|\left(\dot{E}^{\circ} \backslash C\right)<\frac{1}{2} \varepsilon$ and $|\mu|(U \backslash \bar{E})<\frac{1}{2} \varepsilon$. By Urysohn's Lemma, there exist continuous functions $\varphi, \psi$ : $X \rightarrow[0,1]$ such that $\varphi|C=0, \varphi|(X \backslash \stackrel{\circ}{E})=1$, and $\psi|\bar{E}=0, \psi|(X \backslash U)=1$. Since $\varphi$ and $\psi$ are $Q_{\mu}$-continuous, there exist constants $\alpha, \beta \in(0,1)$ for which $|\mu|\left(\varphi^{-1}(\alpha)\right)$ $=0$ and $|\mu|\left(\psi^{-1}(\beta)\right)=0$ and if $K=\{x \in X: \varphi(x)<\alpha\}$ and $V=\{x \in X: \psi(x)$ $\langle\beta\}$, then $K, V \in \mathbb{Q}_{\mu} ; C \subset K \subset \dot{E} \subset E \subset \bar{E} \subset V \subset U$. So from the inclusion

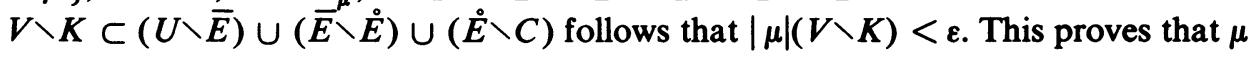
is regular on $\mathbb{Q}_{\mu}$. It now follows that $\mu_{S}$ is regular on $\mathbb{Q}_{\mu} \cap S$.

Since $\int_{S} f|S d| \mu_{S}\left|=\int_{X} f d\right| \mu \mid$ for all $f \in C_{b}(X)$, to identify $\mathbb{Q}_{\mu} \cap S$ it is sufficient to show that $\mathbb{Q}_{\mu}$ consists of all $F \in \mathbb{Q}$ such that

$$
\inf \left\{\int_{X}\left(f_{2}-f_{1}\right) d|\mu|: f_{1} \leqslant \chi_{F} \leqslant f_{2} ; f_{1}, f_{2} \in C_{b}(X)\right\}=0 .
$$

Given $F \in \mathbb{Q}_{\mu}$, then $\stackrel{\circ}{F} \bar{F} \in \mathbb{Q}_{\mu}$. By regularity on $\mathbb{Q}_{\mu}$, given $\varepsilon>0$ there is a closed set $K \in \mathbb{Q}_{\mu}$ and an open set $V \in \mathbb{Q}_{\mu}$ such that $K \subset \stackrel{\circ}{F} \subset F \subset \bar{F} \subset V,|\mu|(K \backslash \dot{F})$ $<\frac{1}{2} \varepsilon$, and $|\mu|(V \backslash \bar{F})<\frac{1}{2} \varepsilon$. By Urysohn's Lemma, there exist continuous functions $\varphi, \psi: X \rightarrow[0,1]$ such that $\varphi|K=1, \varphi|(X \backslash \dot{F})=0$, and $\psi|\bar{F}=1, \psi|(X \backslash V)=0$.

Hence

$$
\begin{aligned}
\int_{X} \varphi d|\mu| & \leqslant \sup \left\{\int_{X} f d|\mu|: f<\chi_{F} ; f \in C_{b}(X)\right\} \\
& <\inf \left\{\int_{X} f d|\mu|: \chi_{F}<f ; f \in C_{b}(X)\right\}<\int_{X} \psi d|\mu|
\end{aligned}
$$

and

$$
\begin{aligned}
\int_{X} \psi d|\mu|-\int_{X} \varphi d|\mu| & =\int_{X}(\psi-\varphi) d|\mu|=\int_{V \backslash K}(\psi-\varphi) d|\mu| \\
& <|\mu|(V \backslash K)=|\mu|(V \backslash \bar{F})+|\mu|(\bar{F} \backslash \stackrel{\circ}{F})+|\mu|(\stackrel{\circ}{F} \backslash K)<\varepsilon
\end{aligned}
$$

imply equation (2.5.1).

Conversely, if $F \notin \mathbb{Q}_{\mu}$, then $|\mu|(\bar{F} \backslash \dot{F})>0$. Choose $\varepsilon>0$ such that $|\mu|(\bar{F} \backslash \dot{F})>$ $\varepsilon$. Given any two continuous functions $\varphi, \psi: X \rightarrow(-\infty, \infty)$ such that $\varphi<\chi_{F}<\psi$, then $\varphi|(\bar{F} \backslash \stackrel{\circ}{F})<0, \psi|(\bar{F} \backslash \stackrel{\circ}{F})>1$ and so $\int_{X} \psi d|\mu|-\int_{X} \varphi d|\mu|>|\mu|(\bar{F} \backslash \dot{F})>\varepsilon$. Thus, if $F \notin \mathbb{Q}_{\mu}$, then equation (2.5.1) does not hold. This completes the proof of the lemma.

2.6. Given a topological space $Y$ and a complex-valued linear functional $\Lambda$ on a subspace $B$ of the Banach space $C_{b}(Y)$ with uniform norm, we write $\|\Lambda\|$ for the norm of $\Lambda$ :

$$
\|\Lambda\|=\sup \{|\Lambda(f)|: f \in B,|f|<1\}
$$

If $\|\Lambda\|<\infty$, then we say that $\Lambda$ is bounded on $B$. We are now in a position to prove the following crucial result. 
2.7 TheOREM. Let $S$ be a dense subset of a compact Hausdorff space $X, \mathbb{Q} \subset$ $\mathscr{B}(X)$ an algebra such that $f \mid S$ is $\mathcal{Q}_{\mu} \cap S$-continuous for all $f \in C(X)$ and all $\mu \in M R(\Re(X))$, and $\Lambda$ a complex-valued bounded linear functional on $C(X)$. The class $\mathcal{E}_{\Lambda}$ of sets $E \in \mathbb{Q} \cap S$ such that

$$
\inf _{f_{1}, f_{2}} \sup _{|g|=1}\left\{\left|\Lambda\left(\left(f_{2}-f_{1}\right) g\right)\right|: f_{1}\left|S<\chi_{E}<f_{2}\right| S ; f_{1}, f_{2}, g \in C(X)\right\}=0
$$

is an algebra of sets for which $f \mid S$ is $\mathcal{E}_{\Lambda}$-continuous for all $f \in C(X)$, and there exists a unique regular bounded finitely additive set function $\lambda: \mathcal{E}_{\Lambda} \rightarrow \mathbf{C}$ with

$$
\Lambda(f)=\int_{S} f \mid S d \lambda \quad(f \in C(X))
$$

Moreover,

$$
\|\Lambda\|=|\lambda|(S)
$$

Proof. Step 1. By the Riesz representation theorem, there exists a unique $\mu \in \operatorname{MR}(\Re(X))$ for which

$$
\Lambda(f)=\int_{X} f d \mu \quad(f \in C(X)) .
$$

Let $\lambda=\mu_{s}$. By the second remark in 2.3, if $f \in C(X)$, then

$$
\Lambda(f)=\int_{S} f \mid S d \lambda
$$

Since a compact Hausdorff space is normal, by Lemma 2.5, $\lambda$ is regular on $\mathbb{Q}_{\mu} \cap S$ and $\mathbb{Q}_{\mu} \cap S$ consists of all $E \in \mathbb{Q} \cap S$ such that

$$
\inf \left\{\int_{X}\left(f_{2}-f_{1}\right) d|\mu|: f_{1}\left|S<\chi_{E}<f_{2}\right| S ; f_{1}, f_{2} \in C(X)\right\}=0 .
$$

But $d|\mu|=g d \mu$ for some Borel measurable function $g$ such that $|g|=1[12, p$. 126]. Hence $\mathbb{Q}_{\mu} \cap S$ consists of all $E \in \mathbb{Q} \cap S$ such that

$$
\inf \left\{\int_{X}\left(f_{2}-f_{1}\right) g d \mu: f_{1}\left|S<\chi_{E}<f_{2}\right| S ; f_{1}, f_{2} \in C(X)\right\}=0 \text {. }
$$

But, by Lusin's theorem, any complex Borel measurable function on $X$ can be approximated in $\mu$-measure by functions in $C(X)$. Hence, $\mathbb{Q}_{\mu} \cap S$ consists of all $E \in \mathbb{Q} \cap S$ such that

$$
\inf _{f_{1}, f_{2}} \sup _{|g|=1}\left\{\left|\int_{X}\left(f_{2}-f_{1}\right) g d \mu\right|: f_{1}\left|S<\chi_{E}<f_{2}\right| S ; f_{1}, f_{2}, g \in C(X)\right\}=0
$$

i.e. such that

$$
\inf _{f_{1}, f_{2}} \sup _{|g|=1}\left\{\left|\Lambda\left(\left(f_{2}-f_{1}\right) g\right)\right|: f_{1}\left|S \leqslant \chi_{E}<f_{2}\right| S ; f_{1}, f_{2}, g \in C(X)\right\}=0 .
$$

Let $\mathcal{E}_{\Lambda}=\mathbb{Q}_{\mu} \cap S$.

Step 2. In this step we establish the uniqueness of $\lambda$ on $\mathcal{E}_{\Lambda}$. Clearly, the total variation of the difference $\nu=\lambda-\lambda^{\prime}$ of two bounded finitely additive set functions regular on $\mathcal{E}_{\Lambda}$ is also a bounded finitely additive set function regular on $\mathcal{E}_{\Lambda}$. Thus it is enough to show that, given a nontrivial bounded finitely additive 
nonnegative set function $\nu$ regular on $\mathcal{E}_{\Lambda}$, then $\int_{S} h \mid S d \nu \neq 0$ for some $h \in C(X)$. Since $\nu \neq 0$, there exists $E \in \mathcal{E}_{\Lambda}$ such that $\nu(E)>0$. By regularity, given $\varepsilon$ $<\frac{1}{2} \nu(E)$, there exist continuous functions $f, g: S \rightarrow[0, \infty)$ and $\alpha, \beta>0$ such that if $K=\{x \in S: f(x)<\alpha\}$ and if $V=\{x \in S: f(x)<\beta\}$ then (i) $K, V \in \mathcal{E}_{\Lambda}$, (ii) $K \subset E \subset V$, and (iii) $\nu(V \backslash K)<\varepsilon$. Now if $\bar{K}$ and $\bar{V}$ are respectively the closed and open subsets of $X$ for which $K=\bar{K} \cap S$ and $V=\bar{V} \cap S$, then by Urysohn's Lemma, there exists a continuous function $h: X \rightarrow[0,1]$ such that $h \mid \bar{K}=1$ and $h \mid(X \backslash \bar{V})=0$. Thus

$$
\begin{aligned}
\int_{S} h \mid S d \nu & =\int_{K} h\left|S d \nu+\int_{V \backslash K} h\right| S d \nu=\nu(K)+\int_{V \backslash K} h \mid S d \nu \\
& =\nu(E)-\nu(E \backslash K)+\int_{V \backslash K} h\left|S d \nu>\nu(E)-\nu(E \backslash K)-\int_{V \backslash K} h\right| S d \nu \\
& \geqslant \nu(E)-2 \nu(V \backslash K) \geqslant \nu(E)-2 \varepsilon>0 .
\end{aligned}
$$

Step 3. This final step consists of showing that $\|\Lambda\|=|\lambda|(S)$. By the Riesz representation theorem

$$
\|\Lambda\|=|\mu|(X)=\sup \left\{\left|\int_{X} f d \mu\right|: f \in C(X),|f|<1\right\} .
$$

But if $L(X, \mu)$ is the Banach space of $\mu$-integrable functions with norm $\int_{X}|f| d \mu<$ $\infty$, then $C(X) \subset C\left(X, Q_{\mu}\right) \subset L(X, \mu)$ and, by Lusin's theorem, $C(X)$ is dense in $L(X, \mu)$. Hence

$$
\begin{aligned}
\|\Lambda\| & =\sup \left\{\left|\int_{X} f d \mu\right|: f \in C\left(X, \mathbb{Q}_{\mu}\right),|f|<1\right\} \\
& =\sup \left\{\left|\int_{S} g d \lambda\right|: g \in C\left(S, \mathcal{E}_{\Lambda}\right),|g|<1\right\}=|\lambda|(S) .
\end{aligned}
$$

The theorem is proved.

2.8 REMARK. If $\Lambda$ is a positive linear functional on $C(X)$, then the associated regular set function $\lambda: \delta_{\Lambda} \rightarrow \mathbf{C}$ of Theorem 2.7 is nonnegative. One way to see this is to apply the Riesz representation theorem for positive linear functionals in Step 1 of the proof. Furthermore, $\mathcal{E}_{\Lambda}$ can be chosen to consist of all $E \in \mathbb{Q} \cap S$ such that

$$
\inf \left\{\Lambda\left(f_{2}-f_{1}\right): f_{1}\left|S<\chi_{E}<f_{2}\right| S ; f_{1}, f_{2} \in C(X)\right\}=0 \text {. }
$$

2.9 Corollary. Given a completely regular (Hausdorff) space $Y$ and a bounded linear functional $L$ on $C_{b}(Y)$, let $\mathcal{E}_{L}$ be the class of sets $E \in \mathscr{B}(Y)$ such that

$$
\inf _{f_{1}, f_{2}} \sup _{|g|=1}\left\{\left|L\left(\left(f_{2}-f_{1}\right) g\right)\right|: f_{1}<\chi_{E}<f_{2} ; f_{1}, f_{2}, g \in C(X)\right\}=0 .
$$

$\mathcal{E}_{L}$ is an algebra of sets for which (i) $f$ is $\mathcal{E}_{L}$-continuous for all $f \in C_{b}(Y)$, and (ii) there exists a unique regular bounded finitely additive complex-valued set function $\lambda$ on $\varepsilon_{L}$ with

$$
L(f)=\int_{Y} f d \lambda \quad\left(f \in C_{b}(Y)\right)
$$

Moreover,

$$
\|L\|=|\lambda|(Y)
$$


Proof. Let $X$ be the Stone-Čech compactification of $Y$. Then $L$ admits a unique (norm-preserving) bounded linear extension $\Lambda: C(X) \rightarrow C$. Taking $S=Y$ and $\mathbb{Q}=\mathscr{B}(X)$ in Theorem 2.7, and noting that, for any $\mu \in M R(\mathscr{B}(X))$ and any $f \in C(X), f \mid S$ is $\mathbb{Q}_{\mu} \cap S$-continuous since the set $\left\{\omega \in \mathbf{C}: \mu\left(f^{-1}(\omega)\right) \neq 0\right\}$ is at most countable, we get the corollary.

2.10 REMARK. In [6, pp. 207, 210-211] sufficient conditions are given for a linear functional on $C(Y)$ ( $Y$ completely regular (Hausdorff)) to admit an integral representation by means of a countably additive measure on $Y$.

2.11. Given a uniform space $Y$, let $U C_{b}(Y)$ denote the Banach space of complex-valued uniformly continuous bounded functions on $Y$ with uniform norm. We obtain a compactification for $Y$ by the following well-known construction. Let $\left\{\varphi_{y}: y \in Y\right\}$ be those linear functionals on $U C_{b}(Y)$ given by $\varphi_{y}(f)=f(y)$. Then the weak closure $X$ of $\left\{\varphi_{y}: y \in Y\right\}$ in the dual of $U C_{b}(Y)$ is weakly compact and we can view $Y$ as a dense subset of $X$ such that $U C_{b}(Y)=\{f \mid Y: f \in C(X)\}$. Thus, Theorem 2.7 yields the following.

2.12 CoROllary. Given a uniform space $Y$ and a bounded linear functional $L$ on $U C_{b}(Y)$, let $\mathcal{E}_{L}$ be the class of sets $E \in \mathscr{B}(Y)$ such that

$$
\inf _{f_{1}, f_{2}} \sup _{|g|=1}\left\{\left|L\left(\left(f_{2}-f_{1}\right) g\right)\right|: f_{1} \leqslant \chi_{E} \leqslant f_{2} ; f_{1}, f_{2}, g \in U C_{b}(Y)\right\}=0 .
$$

$\mathcal{E}_{L}$ is an algebra of sets for which (i) $f$ is $\mathcal{E}_{L}$-continuous for all $f \in U C_{b}(Y)$ and (ii) there exists a unique regular bounded finitely additive complex-valued set function $\lambda$ on $\mathcal{E}_{L}$ with

$$
L(f)=\int_{Y} f d \lambda \quad\left(f \in U C_{b}(Y)\right)
$$

Moreover,

$$
\|L\|=|\lambda|(Y)
$$

\section{Linear functionals on almost periodic functions.}

3.1. Let $G$ be an Abelian locally compact group with dual group $G^{\wedge}$ and Bohr compactification $\bar{G}\left(\left[8\right.\right.$, p. 137], [5, §24], [13, p. 30]). The value of an element $\hat{z} \in G^{\wedge}$ at the point $z \in G$ will be denoted by $\langle z, \hat{z}\rangle$ or $\hat{z}(z)$. Let $A P(G)$ be the space of continuous almost periodic functions on $G$ with uniform norm. By a well-known theorem of harmonic analysis $[8$, p. 168] every element of $A P(G)$ is the restriction on $G$ of a unique element of $C(\bar{G})$. Since $G^{\wedge} \subset A P(G)$, any given $\hat{z} \in G^{\wedge}$ can be extended to a unique continuous function on $\bar{G}$ with values in $T=\{\omega \in C$ : $|\omega|=1\}$. This extension shall be denoted by the same symbol, i.e. by $\hat{z}$. Let $m$ denote the normalized Haar measure on $\mathscr{B}(\bar{G})$.

3.2 LEMMA. If $Q$ is the algebra of sets in $\mathscr{B}(\bar{G})$ generated by sets of the form $\left\{z \in \bar{G}: \hat{z}(z) \in T_{0}\right\}$ for $\hat{z} \in G^{\wedge}$ and $T_{0}$ an arc on $T$ with $m\left(\left\{\hat{z}^{-1}\left(\bar{T}_{0} \backslash \dot{T}_{0}\right)\right\}\right)=0$, then $f \mid G$ is $\mathbb{Q}_{\mu} \cap G$-continuous for all $f \in C(\bar{G})$ and all $\mu \in M R(\mathscr{B}(\bar{G}))$.

Proof. Given $\varepsilon>0$, by the Stone-Weierstrass theorem there exists a polynomial $p(z)$ on $\bar{G}$ such that $|f(z)-p(z)|<\varepsilon / 3$ for all $z \in \bar{G}$. Since every finite linear 
combination of $\mathbb{Q}_{\mu} \cap G$-continuous functions is $\mathcal{Q}_{\mu} \cap G$-continuous and since $p(z)$ is a finite linear combination of characters, which are clearly $\mathcal{Q}_{\mu} \cap G$-continuous, it follows that there exists a finite $\mathbb{Q}_{\mu} \cap G$-measurable partition $\Pi$ of $G$ such that, for all $E \in \Pi$, we have $\left|p(z)-p\left(z^{\prime}\right)\right|<\varepsilon / 3$ for all $z, z^{\prime} \in E$. So from the inequality $\left|f(z)-f\left(z^{\prime}\right)\right| \leqslant|f(z)-p(z)|+\left|p(z)-p\left(z^{\prime}\right)\right|+\left|p\left(z^{\prime}\right)-f\left(z^{\prime}\right)\right|$ follows that $\mid f(z)-$ $f\left(z^{\prime}\right) \mid<\varepsilon\left(z, z^{\prime} \in E\right)$. The lemma is proved.

This lemma now permits us to generalize a concrete representation theorem due to Hewitt [4, pp. 307-308] for bounded linear functionals on $A P(G)$. We note that our algebra is smaller than his and depends on the linear functional in question.

3.3 Theorem. Given a locally compact Abelian group $G$ with dual group $G^{\wedge}$ and given a bounded linear functional $L: A P(G) \rightarrow \mathrm{C}$, let $\mathrm{A}$ be the algebra of subsets of $G$ generated by sets of the form $\left\{z \in G: \hat{z}(z) \in T_{0}\right\}$ for $\hat{z} \in G^{\wedge}$ and $T_{0}$ an arc on $T$ with $m\left(\left\{z \in \bar{G}: \hat{z}(z) \in \bar{T}_{0} \backslash \dot{T}_{0}\right\}\right)=0$ and let $\mathcal{E}_{L}$ be the class of sets $E \in \mathbf{A}$ such that

$$
\inf _{f_{1}, f_{2}} \sup _{|g|=1}\left\{\left|L\left(\left(f_{2}-f_{1}\right) g\right)\right|: f_{1} \leqslant \chi_{E}<f_{2} ; f_{1}, f_{2}, g \in A P(G)\right\}=0 .
$$

$\mathcal{E}_{L}$ is an algebra for which (i) $f$ is $\mathcal{E}_{L}$-continuous for all $f \in A P(G)$ (or equivalently, $\hat{z}$ is $\mathcal{E}_{L}$-continuous for all $\hat{z} \in G^{\wedge}$ ) and (ii) there exists a unique regular bounded finitely additive complex-valued set function $\lambda$ on $\mathcal{E}_{L}$ with

$$
L(f)=\int_{G} f d \lambda \quad(f \in A P(G)) .
$$

Moreover,

$$
\|L\|=|\lambda|(G)
$$

Proof. Recall that a function lies in $A P(G)$ if and only if it is the restriction on $G$ of a (unique) function in $C(\bar{G})[8$, p. 168]. Hence, there exists a bounded linear functional $\Lambda$ on $C(\bar{G})$ given by $\Lambda(h)=L(h \mid G)$. Let $\mathcal{Q}$ be the algebra of sets of Lemma 3.2. Then $A=\mathbb{Q} \cap G$. To complete the proof, apply Theorem 2.7 for $X=\bar{G}, S=G$, and $\mathcal{E}_{\Lambda}=\mathcal{E}_{L}$.

3.4. Given $f \in C(\bar{G})$, write $\hat{f}: G^{\wedge} \rightarrow \mathbf{C}$ for its Fourier transform

$$
\hat{f}(\hat{z})=\int_{\bar{G}} f(z) \overline{\langle z, \hat{z}\rangle} d m(z) .
$$

Let $F(G)$ denote the family of finite linear combinations of characters. By Bohr's fundamental theorem (or equivalently, the Stone-Weierstrass theorem on the Bohr compactification), $F(G)$ is dense in $A P(G)$ with respect to the uniform norm.

3.5 Definition. A complex-valued function $p(\hat{z})$ defined on $G^{\wedge}$ is said to be positive definite if

$$
\sum_{i, j=1}^{n} p\left(\hat{z}_{i}-\hat{z}_{j}\right) c_{i} \bar{c}_{j}>0
$$

for all $\hat{z}_{1}, \ldots, \hat{z}_{n} \in G^{\wedge}$ and for all $c_{1}, \ldots, c_{n} \in \mathbf{C}$.

The following is a generalization of Hewitt's representation of positive definite functions [4, pp. 310-311]. 
3.6 THEOREM. Given a locally compact Abelian group $G$ with dual group $G^{\wedge}$ and given a complex-valued function $p(\hat{z})$ on $G^{\wedge}$, let $\mathbf{A}$ be the algebra of subsets of $G$ generated by sets of the form $\left\{z \in G: \hat{z}(z) \in T_{0}\right\}$ for $\hat{z} \in G^{\wedge}$ and $T_{0}$ an arc on $T$ with $m\left(\left\{z \in \bar{G}: \hat{z}(z) \in \bar{T}_{0} \backslash \dot{T}_{0}\right\}\right)=0$, and let $\mathcal{E}_{p}$ be the class of sets $E \in \mathbf{A}$ such that

$$
\inf \left\{\left|\sum_{\hat{z} \in G^{-}}\left(\hat{f}_{2}(\hat{z})-\hat{f}_{1}(\hat{z})\right) p(\hat{z})\right|: f_{1} \leqslant \chi_{E} \leqslant f_{2} ; f_{1}, f_{2} \in F(G)\right\}=0 .
$$

The function $p(\hat{z})$ is positive definite on $G^{\wedge}$ if and only if (i) $\mathcal{E}_{p}$ is an algebra such that $f$ is $\mathcal{E}_{p}$-continuous for all $f \in A P(G)$ (or equivalently, $\hat{z}$ is $\mathcal{E}_{p}$-continuous for all $\left.\hat{z} \in G^{\wedge}\right)$ and (ii) there exists a unique regular bounded finitely additive nonnegative set function $\lambda$ on $\mathcal{E}_{p}$ such that

$$
p(\hat{z})=\int_{G}\langle z, \hat{z}\rangle d \lambda(z)
$$

for all $\hat{z} \in G^{\wedge}$.

Proof. Sufficiency is easily shown. Necessity can be established by repeating the ideas behind the proof of Bochner's theorem as found in [13, pp. 19-21]. The proof that we now provide is somewhat shorter.

Clearly, a function lies in $F(G)$ if and only if it is the restriction on $G$ of a (unique) function in $F(\bar{G})$. Also, since $|p(z)|<p(0)[13, \mathrm{p} .19], p(z)$ is uniformly bounded. Assume $p(0)=1$. On $F(G)$, define the linear functional $\Lambda$ by

$$
\Lambda(f \mid G)=\sum_{\hat{z} \in G^{-}} \hat{f}(\hat{z}) p(\hat{z}) \quad(f \in F(\bar{G})) .
$$

Let $\hat{F}(\bar{G})=\{\hat{f}: f \in F(\bar{G})\}$ and given $f, g \in F(\bar{G})$, put

$$
(\hat{f}, \hat{g})=\Lambda(f \bar{g} \mid G) \text {. }
$$

This defines an inner product on $\hat{F}(\bar{G})$ and so $|\Lambda(f \bar{g} \mid G)|^{2}<\Lambda\left(|f|^{2} \mid G\right) \Lambda\left(|g|^{2} \mid G\right)$. Thus,

$$
\begin{aligned}
|\Lambda(f \mid G)|^{2} & \leqslant \Lambda\left(|f|^{2} \mid G\right) \leqslant \Lambda\left(\left|f^{2}\right|^{2} \mid G\right)^{1 / 2}<\cdots<\Lambda\left(\left|f^{2}\right|^{2^{n}} \mid G\right)^{2^{-n}}=\Lambda\left(\left|f^{2^{n}}\right|^{2} \mid G\right)^{2^{-n}} \\
& =\left(\sum_{\hat{z}} \sum_{\hat{w}}\left(f^{2^{n}}\right)^{-}(\hat{z})\left(f^{2^{n}}\right)^{\hat{i}}(\hat{w}) p(\hat{z}-\hat{w})\right)^{2^{-n}} \\
& \leqslant\left(\sum_{\hat{z}}\left|\left(f^{2^{n}}\right)^{\wedge}(\hat{z})\right|\right)^{2^{-n+1}}=\left(\int_{\bar{G}}\left|f^{2^{n-1}}\right|^{2} d m\right)^{2^{-n+1}} \\
& \rightarrow \sup \left\{|f(z)|^{2}: z \in \bar{G}\right\} \quad(n \rightarrow \infty) .
\end{aligned}
$$

Thus $\Lambda$ is a bounded linear functional on $F(G)$ and so can be extended to a unique bounded linear functional $\Lambda^{\prime}$ on the closure $A P(G)$ of $F(G)$ with respect to the uniform norm. Note that $\left\|\Lambda^{\prime}\right\|=1$. Thus, by Theorem 3.3, there exist an algebra $\mathcal{E} \subset \mathbf{A}$ and a unique regular finitely additive complex-valued set function $\lambda$ on $\mathcal{E}$ such that

$$
\Lambda^{\prime}(f)=\int_{G} f d \lambda \quad(f \in A P(G))
$$


and

$$
\left\|\Lambda^{\prime}\right\|=|\lambda|(G)
$$

In particular, if $f(z)=\langle z, \hat{z}\rangle$, then

$$
p(\hat{z})=\int_{G}\langle z, \hat{z}\rangle d \lambda(z)
$$

for all $\hat{z} \in G^{\wedge}$. Furthermore, since $1=p(0)=\int_{G} d \lambda=\lambda(G) \leqslant|\lambda|(G)=\left\|\Lambda^{\prime}\right\|=1$, then $\lambda$ is nonnegative on $\mathcal{E}$ and by Remark $2.8, \mathcal{E}$ consists of all $E \in \mathbf{A}$ with

$$
\inf \left\{\Lambda^{\prime}\left(f_{2}-f_{1}\right): f_{1} \leqslant \chi_{E} \leqslant f_{2} ; f_{1}, f_{2} \in A P(G)\right\}=0 .
$$

Hence, since $F(G)$ is dense in $A P(G)$ with respect to the uniform norm, equation (3.6.1) identifies $\mathcal{E}$. The theorem is proved.

\section{Fourier-Stieltjes transforms.}

4.1. Given a locally compact Abelian group $G$, let $M(G)$ denote the family of tuples $(\mu, \mathbb{Q})$ where $\mathcal{Q}$ is an algebra of sets in $\mathscr{B}(G)$ such that each $f \in A P(G)$ (or equivalently, each character $\hat{z} \in G^{\wedge}$ ) is $Q$-continuous and $\mu$ is a bounded finitely additive complex-valued set function on $Q$. Recall that the Fourier-Stieltjes transform $\hat{\mu}$ of $(\mu, \mathcal{Q})$ is given by $\hat{\mu}(\hat{z})=\int_{G}\langle z, \hat{z}\rangle d \mu(z)$. Write $B\left(G^{\wedge}\right)$ for the space of all finite linear combinations of positive definite functions on $G^{\wedge}$. By the Pontryagin duality theorem, the group $G$ can be identified with $G^{\hat{\hat{n}}}$ and so, if $z \in G$, the character $\hat{z} \rightarrow\langle z, \hat{z}\rangle$ lies in $B\left(G^{\wedge}\right)$. Also, given $(\mu, \mathcal{Q}) \in M(G)$, then $\mu=\left(\mu_{1}^{+}-\right.$ $\left.\mu_{1}^{-}\right)+i\left(\mu_{2}^{+}-\mu_{2}^{-}\right)$where $\mu_{j}^{ \pm}(j=1,2)$ are nonnegative bounded set functions on $\mathbb{Q}\left[15\right.$, p. 401]. So, it is easy to see that $\hat{\mu} \in B\left(G^{\wedge}\right)$. In fact, we have the following.

4.2 Lemma. A complex-valued function on $G^{\wedge}$ lies in $B\left(G^{\wedge}\right)$ if and only if it is the Fourier-Stieltjes transform of an element of $M(G)$.

Proof. It remains to prove necessity. Let $p \in B\left(G^{\wedge}\right)$. Then $p(\hat{z})=\Sigma_{k} p_{k}(\hat{z})$ where the sum is finite and $p_{k}$ is a scalar multiple of a positive definite function on $G$. So, by Theorem 3.6, there exist $\left(\mu_{k}, \mathbb{Q}_{k}\right) \in M(G)$ for all $k$, such that, $p_{k}(\hat{z})=$ $\int_{G}\langle z, \hat{z}\rangle d \mu_{k}(z)$. Let $\Lambda_{k}$ be those bounded linear functionals on $A P(G)$ given by $\Lambda_{k}(f)=\int_{G} f d \mu_{k}$ and put $\Lambda=\Sigma_{k} \Lambda_{k}$ on $A P(G)$. Then, by Theorem 3.3, there exists $(\mu, \mathcal{E}) \in M(G)$ such that $\Lambda(f)=\int_{G} f d \mu(f \in A P(G))$ and so, if $g(z)=\langle z, \hat{z}\rangle$, then

$$
\hat{\mu}(\hat{z})=\Lambda(g)=\sum_{k} \Lambda_{k}(g)=\sum_{k} p_{k}(\hat{z})=p(\hat{z}) .
$$

The lemma is proved.

4.3. Since a function lies in $B\left(G^{\wedge}\right)$ if and only if it is the Fourier-Stieltjes transform $\hat{\mu}$ of a particular $(\mu, \mathbb{Q}) \in M(G)$ given uniquely by Theorem 3.6, we define a norm on $B\left(G^{\wedge}\right)$ by $\|\hat{\mu}\|=|\mu|(G)$. We can now prove the following analog for $B\left(G^{\wedge}\right)$ of Eberlein's characterization of $B\left(G^{\wedge}\right) \cap C\left(G^{\wedge}\right)$ (see [13, pp. 32-34]).

4.4 THEOREM. A function $p$ lies in $B\left(G^{\wedge}\right)$ if and only if

$$
\left|\sum_{G^{*}} \hat{f}(\hat{z}) p(\hat{z})\right| \leqslant\|p\| \sup \{|f(z)|: z \in G\}
$$

for all $f \in F(G)$. 
Proof. Suppose $p \in B\left(G^{\wedge}\right)$. By Theorem 3.6, there exists $(\lambda, \mathcal{E}) \in M(G)$ such that $p(\hat{z})=\int_{G}\langle z, \hat{z}\rangle d \lambda(z)$. Hence $\|p\|=|\lambda|(G)$. Let $\Lambda$ be the linear operator on $A P(G)$ given by $\Lambda(f)=\int_{G} f d \lambda$. Then $\Lambda(f)=\Sigma_{G} \hat{f}(\hat{z}) p(\hat{z})$ for all $f \in F(G)$. Thus, since $\|\Lambda\|=|\lambda|(G)=\|p\|$ and $|\Lambda(f)| \leqslant\|\Lambda\| \sup \{|f(z)|: z \in G\}$, then (4.4.1) holds for all $f \in F(G)$.

Conversely, suppose that (4.4.1) holds for all $f \in F(G)$. Let $\Lambda$ be the linear functional on $F(G)$ defined by $\Lambda(f)=\Sigma_{G} \hat{f}(\hat{z}) p(\hat{z})$. Then, by (4.4.1), $\Lambda$ is bounded by $\|p\|$ on $F(G)$. Let $\Lambda^{\prime}$ be the (unique) linear functional extending $\Lambda$ to the closure $A P(G)$ of $F(G)$ with respect to the uniform norm. Then $\left\|\Lambda^{\prime}\right\| \leqslant\|p\|$. By Theorem 3.3, there exists a tuple $(\lambda, \mathcal{E}) \in M(G)$ such that $\Lambda^{\prime}(f)=\int_{G} f d \lambda$ for all $f \in$ $A P(G)$. So, given $\hat{z} \in G^{\wedge}$, if $g(z)=\langle z, \hat{z}\rangle$, then $\hat{\lambda}(\hat{z})=\int_{G} g d \lambda=\Lambda^{\prime}(g)=\Lambda(g)=$ $p(\hat{z})$. Since $\hat{\lambda} \in B\left(G^{\wedge}\right)$, then $p \in B\left(G^{\wedge}\right)$ and this completes the proof of the theorem.

4.5 COROllary. Given a pointwise convergent net in $B\left(G^{\wedge}\right)$ uniformly bounded in norm by some constant, the limit also lies in $B\left(G^{\wedge}\right)$ and is bounded in norm by the same constant.

Proof. This follows immediately from (4.4.1).

4.6. Let $X$ and $Y$ be arbitrary sets and let $f: X \times Y \rightarrow$ C. We say that $f$ satisfies the double limit condition if, whenever $\left(x_{i}\right)$ and $\left(y_{i}\right)$ are sequences in $X$ and $Y$ respectively such that the iterated limits $\alpha=\lim _{i \rightarrow \infty} \lim _{j \rightarrow \infty} f\left(x_{i}, y_{j}\right)$ and $\beta=$ $\lim _{j \rightarrow \infty} \lim _{i \rightarrow \infty} f\left(x_{i}, y_{j}\right)$ exist, then $\alpha=\beta$. In particular, for a locally compact Abelian group $G$, the function $f: G \times G^{\wedge} \rightarrow T$ given by $f(z, \hat{z})=\langle z, \hat{z}\rangle$ satisfies the double limit condition. To see this, we first note that the restriction to $G$ of an element of $\overline{(\bar{G})^{\wedge}}$ (also denoted $G^{-\wedge-}$ ) belongs to $\overline{G^{\wedge}}$ (also denoted $G^{\wedge-}$ ). From this follows that the restriction to $G^{-\wedge}$ of an element of $G^{-\wedge-\wedge-}$ belongs to $G^{-}$. Note also that $G$ and $G^{\wedge}$ with discrete topology are topological subgroups of $G^{-\wedge-\wedge}$ and $G^{-\wedge \cdots}$ respectively. Hence, if $\left(z_{i}\right)$ and $\left(\hat{z}_{j}\right)$ are sequences in $G$ and $G^{\wedge}$ respectively, then there are subsequences $\left(z_{i m}\right)$ and $\left(\hat{z}_{j n}\right)$ of $\left(z_{i}\right)$ and $\left(\hat{z}_{j}\right)$ respectively such that $\lim _{m \rightarrow \infty} \lim _{n \rightarrow \infty}\left\langle z_{i m}, \hat{z}_{j n}\right\rangle=\lim _{n \rightarrow \infty} \lim _{m \rightarrow \infty}\left\langle z_{i m}, \hat{z}_{j n}\right\rangle$. From this follows that the function $f(z, \hat{z})=\langle z, \hat{z}\rangle$ satisfies the double limit condition.

An algebra $\mathcal{Q}$ of sets in $X$ is said to separate points on $X$ if, whenever $x, y \in X$, $x \neq y$, there are disjoint sets $A, B \in \mathbb{Q}$ such that $x \in A$, and $y \in B$. Clearly, if $\mathbb{Q}$ is a subalgebra of $\mathscr{B}(G)$ such that each $f \in A P(G)$ is $Q$-continuous, then by Urysohn's Lemma and the Stone-Weierstrass theorem on $\bar{G}$, it follows that $\mathbb{Q}$ separates points on $G$. Now, by a theorem of Sinclair [14, pp. 363, 364], if $\mathscr{Q}$ and $\mathcal{E}$ are algebras of sets which separate points on $X$ and $Y$ respectively and if $f$ : $X \times Y \rightarrow \mathbf{C}$ is a bounded function for which (i) $f(\cdot, y)$ is $\mathbb{Q}$-continuous for all $y \in Y$, (ii) $f(x, \cdot)$ is $\mathcal{E}$-continuous for all $x \in X$, and (iii) $f$ satisfies the double limit condition, then (1) $\int_{X} f(x, y) d \mu(x)$ and $\int_{Y} f(x, y) d \lambda(y)$ are integrable with respect to $\mu$ and $\lambda$ respectively and (2) $\int_{X} \int_{Y} f d \lambda d \mu=\int_{Y} \int_{X} f d \mu d \lambda$, for all finitely additive bounded complex-valued set functions $\mu$ and $\lambda$ on $\mathscr{Q}$ and $\mathcal{E}$ respectively. Thus, if $(\mu, \mathcal{Q}) \in M(G)$ and if $(\lambda, \mathcal{E}) \in M\left(G^{\wedge}\right)$, then (1) the Fourier-Stieltjes transforms $\hat{\mu}(\hat{z})=\int_{G}\langle z, \hat{z}\rangle d \mu(z)$ and $\hat{\lambda}(z)=\int_{G}\langle z, \hat{z}\rangle d \lambda(\hat{z})$ are integrable with 
respect to $\lambda$ and $\mu$ respectively, and (2) $\int_{G} \int_{G^{*}}\langle z, \hat{z}\rangle d \lambda(\hat{z}) d \mu(z)=$ $\int_{G^{-}} \int_{G}\langle z, \hat{z}\rangle d \mu(z) d \lambda(\hat{z})$.

4.7 Remark. Let $L\left(G^{\wedge}\right)$ denote the space of complex-valued functions on $G^{\wedge}$ which are integrable with respect to the Haar measure $d \hat{z}$ on $\mathscr{B}\left(G^{\wedge}\right)$. Given $h \in L\left(G^{\wedge}\right)$, write $\tilde{h}$ for the continuous complex-valued function on $G$ given by the Fourier transform $\tilde{h}(z)=\int_{G^{*}}\langle z, \hat{z}\rangle h(\hat{z}) d \hat{z}$. If $p \in \in^{\prime} B\left(G^{\wedge}\right)$, then

$$
\left|\int_{G^{+}} h(\hat{z}) p(\hat{z}) d \hat{z}\right| \leqslant\|p\| \sup \{|\tilde{h}(z)|: z \in G\}
$$

for all $h \in L\left(G^{\wedge}\right)$. This well-known property of Fourier-Stieltjes transforms (see [5, §33.20]) can be proved as follows. Suppose that $p \in B\left(G^{\wedge}\right)$. Then, there exists $(\lambda, \mathcal{E}) \in M(G)$ such that $p(\hat{z})=\int_{G}\langle z, \hat{z}\rangle d \lambda(z)$. Also, for any $h \in L\left(G^{\wedge}\right), h(\hat{z}) d \hat{z}$ is a finitely additive set function, say $\mu$, on $\mathscr{B}\left(G^{\star}\right)$. So, by Sinclair's theorem,

$$
\begin{aligned}
\left|\int_{G^{+}} h(\hat{z}) p(\hat{z}) d \hat{z}\right| & =\left|\int_{G^{*}} p(\hat{z}) d \mu(\hat{z})\right|=\left|\int_{G^{+}} \int_{G}\langle z, \hat{z}\rangle d \lambda(z) d \mu(\hat{z})\right| \\
& =\left|\int_{G} \int_{G^{+}}\langle z, \hat{z}\rangle d \mu(\hat{z}) d \lambda(z)\right|=\left|\int_{G} \int_{G^{+}}\langle z, \hat{z}\rangle h(\hat{z}) d \hat{z} d \lambda(z)\right| \\
& =\left|\int_{G} \tilde{h}(z) d \lambda(z)\right| \leqslant|\lambda|(G) \sup \{|\tilde{h}(z)|: z \in G\} \\
& =\|p\| \sup \{|\tilde{h}(z)|: z \in G\} \quad \text { for all } h \in L\left(G^{\wedge}\right) .
\end{aligned}
$$

4.8. Recall that if $G=T$, then $G^{\wedge}=Z$ where $Z=\{0, \pm 1, \pm 2, \ldots\}$. Let $\left\{a_{k, n}\right.$ : $k, n \in Z\}$ be a matrix of complex numbers such that $\Sigma_{n}\left|a_{k, n}\right|<\infty$ for all $k \in Z$. Then the sums $\Sigma_{n} p(n) a_{k, n}$ are well defined for all $p \in B(Z)$. We wish to give a criterion for the matrix to have the property that, for all $p \in B(Z), \Sigma_{n} p(n) a_{k, n}$ lies in $B(Z)$ as a function of $k$. As the following theorem shows, such a condition is given by $\Sigma_{n} z^{n} a_{k, n} \in B(Z)$ for all $z \in T$.

4.9 TheOREM. Let $G$ be a locally compact Abelian group and let $\left\{L_{\hat{z}}: \hat{z} \in G^{\wedge}\right\}$ be a family of complex-valued linear functionals on $B\left(G^{\wedge}\right)$ bounded with respect to the uniform norm. In order that, for all $(\mu, \mathbb{Q}) \in M(G), \hat{z} \rightarrow L_{\hat{z}}(\hat{\mu})$ be the FourierStieltjes transform of an element of $M(G)$, it is necessary and sufficient that $L_{\hat{z}}(z)$ lie in $B\left(G^{\wedge}\right)$ for each function $z \in G^{\wedge \wedge}$.

Proof. By Theorem 3.3, for each $\hat{z} \in G^{\wedge}$, there exists $\left(\lambda_{\hat{z}}, Q_{\hat{z}}\right) \in M\left(G^{\wedge}\right)$ such that $L_{\hat{z}}(g)=\int_{G^{*}} g(\hat{w}) d \lambda_{\hat{z}}(\hat{w})$ for all $g \in F\left(G^{*}\right)$. Hence the theorem reduces to showing the following: given $\left\{\left(\lambda_{\hat{z}}, \mathbb{Q}_{\hat{z}}\right): \hat{z} \in G^{\wedge}\right\} \subset M\left(G^{\wedge}\right)$, then in order that, for all $(\mu, \mathcal{Q}) \in M(G), \int_{G^{-}} \hat{\mu}(\hat{w}) d \lambda_{\dot{z}}(\hat{w})$ be the Fourier-Stieltjes transform of an element of $M(G)$, it is necessary and sufficient that $\hat{\lambda}_{\dot{z}}(z) \in B\left(G^{\wedge}\right)$ for each $z \in G^{\wedge}$.

To prove necessity, suppose that, for any given $w \in G,(\mu, \mathcal{Q}) \in M(G)$ is such that for any $E \in Q, \mu(E)=1$ if $w \in E$ and $\mu(E)=0$ otherwise. Then, by hypothesis, there exists $(\nu, \mathcal{E}) \in M(G)$ such that 


$$
\begin{aligned}
\hat{\nu}(\hat{z}) & =\int_{G^{\mu}} \hat{\mu}(\hat{w}) d \lambda_{\dot{z}}(\hat{w})=\int_{G^{-}} \int_{G}\langle z, \hat{w}\rangle d \mu(z) d \lambda_{\dot{z}}(\hat{w}) \\
& =\int_{G^{-}}\langle w, \hat{w}\rangle d \lambda_{\hat{z}}(\hat{w})=\hat{\lambda}_{\dot{z}}(w) .
\end{aligned}
$$

But $\nu=\left(\nu_{1}^{+}-\nu_{1}^{-}\right)+i\left(\nu_{2}^{+}-\nu_{2}^{-}\right)$where $\nu_{j}^{ \pm}(j=1,2)$ are nonnegative bounded finitely additive set functions on $\delta\left[15\right.$, p. 401]. So, $\hat{\nu}=\left(\hat{\nu}_{1}^{+}-\hat{\nu}_{1}^{-}\right)+i\left(\hat{\nu}_{2}^{+}-\hat{\nu}_{2}^{-}\right)$ where, by Theorem 3.6, $\hat{\nu}_{j}^{ \pm}(j=1,2)$ are positive definite on $G^{\wedge}$. Hence, for any given $w \in G, \hat{\lambda}_{\dot{z}}(w)$ is a linear combination of four positive definite functions in $\hat{z}$.

We now prove sufficiency. Given $(\mu, \mathcal{Q}) \in M(G)$, there exist bounded nonnegative finitely additive set functions $\mu_{j}^{ \pm}(j=1,2)$ on $\mathbb{Q}$ such that $\mu=\left(\mu_{1}^{+}-\mu_{1}^{-}\right)+$ $i\left(\mu_{2}^{+}-\mu_{2}^{-}\right)$. Hence, for $j=1,2$,

$$
\begin{aligned}
\int_{G^{-}} \hat{\mu}_{J}^{ \pm}(\hat{w}) d \lambda_{\bar{z}}(\hat{w}) & =\int_{G^{-}} \int_{G}\langle z, \hat{w}\rangle d \mu_{j}^{ \pm}(z) d \lambda_{\dot{z}}(\hat{w}) \\
& =\int_{G} \int_{G^{-}}\langle z, \hat{w}\rangle d \lambda_{\bar{z}}(\hat{w}) d \mu_{j}^{ \pm}(z)=\int_{G} \hat{\lambda}_{\dot{z}}(z) d \mu_{j}^{ \pm}(z) .
\end{aligned}
$$

Thus, by hypothesis on $\hat{\lambda}_{\dot{z}}(z), \int_{G^{\star}} \hat{\mu}_{j}^{ \pm}(\hat{w}) d \lambda_{\dot{z}}(\hat{w})$ lies in $B\left(G^{\wedge}\right)$. So, by Lemma 4.2 , there exist elements $\left(\nu_{j}^{ \pm}, Q_{j}^{ \pm}\right) \in M(G)(j=1,2)$ such that $\hat{\nu}_{j}^{ \pm}(\hat{z})=$ $\int_{G^{-}} \hat{\mu}_{j}^{ \pm}(\hat{w}) d \lambda_{\dot{z}}(\hat{w})$. Let $\Lambda_{j}^{ \pm}(j=1,2)$ be those bounded complex-valued linear functionals on $A P(G)$ given by $\Lambda_{j}^{ \pm}(f)=\int_{G} f(z) d \nu_{j}^{ \pm}(z)$ and, on $A P(G)$, put $\Lambda=$ $\left(\Lambda_{1}^{+}-\Lambda_{1}^{-}\right)+i\left(\Lambda_{2}^{+}-\Lambda_{2}^{-}\right)$. Then, by Theorem 3.3 , there exists $(\nu, \delta) \in M(G)$ such that $\Lambda(f)=\int_{G} f(z) d \nu(z)$ for all $f \in A P(G)$, and so $\hat{\nu}(\hat{z})=\Lambda(\hat{z})=$ $\int_{G^{\wedge}} \hat{\mu}(\hat{w}) d \lambda_{\hat{z}}(\hat{w})$. The theorem is proved.

4.10 Corollary. If $(\lambda, \mathcal{E}) \in M\left(G^{\wedge}\right)$, then, for all $(\mu, \mathbb{Q}) \in M(G)$,

$$
\int_{(i} \hat{\mu}(\hat{z}-\hat{w}) d \lambda(\hat{w})
$$

is the Fourier-Stieltjes transform of an element of $M(G)$.

Proof. Let $\left\{L_{\hat{z}}: \hat{z} \in G^{\wedge}\right\}$ be that family of bounded complex-valued linear functionals on $B\left(G^{\hat{A}}\right)$ given by $L_{\hat{z}}(f)=\int_{G} f(\hat{z}-\hat{w}) d \lambda(\hat{w})$. Then, for $g(\hat{z})=\langle z, \hat{z}\rangle$ where $z$ is given in $G, L_{\hat{z}}(g)=\int_{G^{\cdot}}\langle z, \hat{z}-\hat{w}\rangle d \lambda(\hat{w})=\langle z, \hat{z}\rangle \hat{\lambda}(-z) \in B\left(G^{\wedge}\right)$. So, the corollary follows from Theorem 4.9.

\section{REFERENCES}

1. R. B. Darst, A note on abstract integration, Trans. Amer. Math. Soc. 99 (1961), 292-297.

2. N. Dunford and J. T. Schwartz, Linear operators, Part I, Interscience, New York, 1958.

3. U. an der Heiden, On the representation of linear functionals by finitely additive set functions, Arch. Math. 30 (1978), 210-214.

4. E. Hewitt, Linear functionals on almost periodic functions, Trans. Amer. Math. Soc. 74 (1953), 303-322.

5. E. Hewitt and K. A. Ross, Abstract harmonic analysis. I, II, Springer-Verlag, New York, 1963.

6. S. S. Khurana, Lattice valued Borel measures. II, Trans. Amer. Math. Soc. 235 (1978), 205-211.

7. S. Leader, On universally integrable functions, Proc. Amer Math. Soc. 6 (1955), 232-234.

8. L. H. Loomis, An introduction to harmonic analysis, Van Nostrand, New York, 1953.

9. __ Linear functionals and content, Amer. J. Math. 76 (1954), 168-182.

10. D. Pollard and F. Topsee, A unified approach to Riesz type representation theorems, Studia Math. S4 (1975), 173-190. 
11. P. C. Rosenbloom, Quelques classes de problèmes extrémaux, Bull. Soc. Math. France 80 (1952), 183-215.

12. W. Rudin, Real and complex analysis, McGraw-Hill, New York, 1966.

13. Fourier analysis on groups, Interscience, New York, 1967.

14. G. E. Sinclair, A finitely additive generalization of the Fichtenholz-Lichtenstein theorem, Trans. Amer. Math. Soc. 193 (1974), 359-374.

15. A. Taylor, Introduction to functional analysis, Wiley, New York, 1958.

16. F. Topsce, Further results on integral representations, Studia Math. 55 (1976), 239-245.

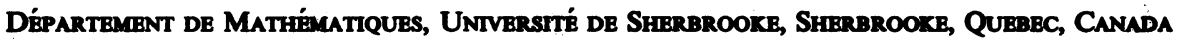

